\title{
ZAP-70 constitutively regulates gene expression and protein synthesis in chronic lymphocytic leukemia
}

\author{
Jingyu Chen ${ }^{1,2}$, Vijitha Sathiaseelan ${ }^{1,2}$, Andrew Moore ${ }^{1,2}$, Shengjiang Tan ${ }^{1,2,6}$, Chandra \\ Sekkar Reddy Chilamakuri³, Valar Nila Roamio Franklin ${ }^{3}$, Arash Shahsavari ${ }^{1}$, Constanze \\ Jakwerth $^{4}$, Sandra B. Hake ${ }^{5}$, Alan J. Warren ${ }^{1,2,6}$, Irina Mohorianu ${ }^{1}$, Clive D'Santos ${ }^{3}$ and Ingo \\ Ringshausen ${ }^{1,2}$
}

1. Wellcome / MRC Cambridge Stem Cell Institute, Jeffrey Cheah Biomedical Centre, University of Cambridge, Cambridge CB2 OAW, United Kingdom.

2. Department of Haematology, University of Cambridge, Cambridge CB2 OAH, United Kingdom.

3. Cancer Research UK Cambridge Institute, University of Cambridge, Cambridge, United Kingdom.

4. Center for Allergy and Environment (ZAUM), Technical University and Helmholtz Center Munich, Munich, Germany; Member of the German Center of Lung Research (DZL),

5. Institute for Genetics, Justus-Liebig University Giessen, 35392, Giessen, Germany

6. Cambridge Institute for Medical Research, The Keith Peters Building, University of Cambridge, Hills Road, Cambridge CB2 OXY

Abstract word count: 207

Running title: Constitutive functions of ZAP-70 in CLL

\section{Corresponding author:}

Ingo Ringshausen M.D.

University of Cambridge

Wellcome/ MRC Cambridge Stem Cell Institute \& Department of Haematology

Jeffrey Cheah Biomedical Centre

Cambridge Biomedical Campus

Puddicombe Way

CB2 OAW Cambridge

United Kingdom

Phone: +44 1223762086

Email: ir279@cam.ac.uk

\section{Key points:}

1. ZAP-70 is essential to maintain a constitutive survival signal for CLL cells and is directly involved in the expression of CCL3 and CCL4.

2. Upon BCR-activation ZAP70 increasingly forms complexes with ribosomes and promotes MYC-expression and protein synthesis. 


\section{ABSTRACT}

The expression of ZAP-70 in a subset of CLL patients strongly correlates with a more aggressive clinical course, though the exact underlying mechanisms remain elusive. The ability of ZAP-70 to enhance B cell receptor (BCR) signaling, independently of its kinase function, is considered to contribute. Here we employed RNA-sequencing and proteomic analyses of primary cells differing only in their expression of ZAP-70 to further define how ZAP-70 increases aggressiveness of CLL. We identified that ZAP-70 is directly required for cell survival in the absence of an overt BCR signal, which can compensate for ZAP-70 deficiency as an anti-apoptotic signal. In addition, the expression of ZAP-70 regulates the transcription of factors regulating recruitment and activation of T cells, such as CCL3, CCL4 and IL4I1. Quantitative mass spectrometry of double-cross linked ZAP-70 complexes further demonstrated constitutive and direct protein-protein interactions between ZAP-70 and BCRsignaling components. Unexpectedly, ZAP-70 also binds to ribosomal proteins, which is not dependent on, but further increased by BCR-stimulation. Importantly, decreased expression of ZAP-70 significantly reduced MYC-expression and global protein synthesis, providing evidence that ZAP-70 contributes to translational dysregulation in CLL. In conclusion, ZAP-70 constitutively promotes cell survival, microenvironment-interactions and protein synthesis in CLL cells, likely to improve cellular fitness and to further drive disease progression. 


\section{INTRODUCTION}

The tyrosine kinase ZAP-70 is an essential molecule for upstream T cell receptor signaling, required for cell-activation and development ${ }^{1,2}$. Its expression in malignant $B$ cells of a subset of patients with CLL (thereafter named ZAP-70pos) has been shown to correlate with unmutated IGHV genes ${ }^{3,4}$. It was therefore not surprising that its presence is predictive of a more aggressive clinical course. However, analyses of discordant cases demonstrated that it is an independent biomarker and possesses stronger predictive power than the IGHV status ${ }^{5}$. Ultimately, this also suggested that ZAP-70 has a biological function relevant to disease progression. In several studies, it was demonstrated that the presence of ZAP-70 enhances $B C R$-signaling induced by anti-lgM stimulation, though tyrosine kinase activity appears to be dispensable for this ${ }^{6-8}$. Since the presence of a BCR-activating (auto)-antigen seems to be a feature predominantly of M-CLL (and ZAP-70 neg) based on the expression of slgM ${ }^{9}$, it remains a conundrum when precisely this function of ZAP-70 becomes relevant in vivo.

Several studies have described properties of ZAP-70pos cells which are likely relevant to the pathogenesis of the disease, such as increased secretion of CCL3 and CCL4 ${ }^{10}$. In addition, ZAP-70 expression is associated with an increased migration potential ${ }^{11-13}$. These studies provided valuable information to better understand the biology of ZAP-70 positive CLL. However, due to the correlative nature of these studies, it remains largely unknown whether ZAP-70 is a biomarker indicating the presence of these properties, mediated by other factors, or whether ZAP-70 actively contributes. The difficulties inherent to manipulating primary CLL cells were the hitherto major problem to address this question. Therefore, functional studies were often performed only in cell lines, which in several aspects are poor models of CLL. We here provide evidence that ZAP-70 is directly implicated in the provision of survival signals, protein synthesis and recruitment of $T$ cells.

\section{MATERIAL AND METHODS}

\section{Cell culture}

After patients' informed consent and in accordance with the Helsinki Declaration, peripheral blood was obtained from CLL patients. Studies were approved by the Cambridgeshire Research Ethics Committee (07/MRE05/44). Mononuclear cells were isolated from heparinized blood by centrifuging over a Ficoll-Hypaque layer (PAN-Biotech). Cells were further isolated with anti-CD2 and anti-CD14 beads, resulting in 95\%-99\% pure CD5 ${ }^{+}$CD19+ cells. After thawing of cryopreserved samples, cells were cultured in RPMI 1640 (Gibco), supplemented with $10 \%$ fetal bovine serum (FBS), penicillin/streptomycin $50 \mathrm{U} / \mathrm{ml}$, sodium pyruvate $1 \mathrm{mM}$, L-glutamine $2 \mathrm{mM}$, L-asparagine $20 \mathrm{mg} / \mathrm{ml}, 50 \mu \mathrm{M}$ 2-mercaptoethanol, $10 \mathrm{mM}$ HEPES, and MEM non-essential amino acids (Gibco). ZAP-70 status was assessed by flow cytometry using intracellular staining with an anti-ZAP-70 antibody (Becton Dickinson 
Biosciences). Samples with $>\mathbf{2 0} \%$ positive cells compared to isotype control were considered ZAP-70pos.

\section{Nucleofection of primary CLL cells}

Nucleofector technology (Lonza) was used to deliver siRNA to primary cells. $5 \times 10^{6} \mathrm{CLL}$ cells were re-suspended in $100 \mu \mathrm{l}$ Solution-V with $7.5 \mu \mathrm{L}$ of non-silencing (NSC) control siRNA

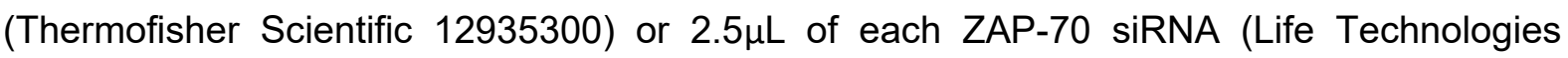
HSS187732, HSS187733, HSS187734). The cell-siRNA suspension was nucleofected using the Nucleofector $2 b$ device (Lonza) (program Cell type-4, X-001). Cell pellets were collected and re-suspended in $3 \mathrm{ml}$ fresh media. siRNA-transfected CLL cells were co-cultured with $5 \times 10^{4}$ stromal cells (plated 24 hours prior to co-culturing CLL cells) per well in a 6-well plate for 7 days.

\section{Pull down of endogenous ZAP-70 from primary CLL cells}

For each condition $20 \times 10^{6}$ purified CLL cells were used. Post BCR-activation using a rabbitanti-human anti-IgM antibody, cells were fixed with DSG (disuccinimidyl glutarate) for 20 minutes at room temperature. Formaldehyde (1\%) in serum-free media was then added for 10 minutes at room temperature, followed by quenching with $125 \mu \mathrm{M}$ glycine for 5 minutes ${ }^{14}$. Cells were then washed by cold 1XPBS twice. Pellets were re-suspended in IP lysis buffer (Thermo Scientific) (supplemented with protease inhibitors) and went through three 'freeze and thaw' cycles on dry ice. Samples were then span at 13000rpm for 6 minutes and supernatant containing the whole cell lysates were collected. An aliquot of supernatant was saved as input for further analysis, the remaining supernatant was incubated overnight at $4^{\circ} \mathrm{C}$ with an anti-ZAP-70 antibody or IgG. Prewashed protein-A beads were added and further incubated for 4 hours at $4^{\circ} \mathrm{C}$. After incubation, the beads were bound to a magnetic rack, then wash buffer removed and processed for mass spectrometry.

\section{Measurement of protein synthesis}

$5 \times 10^{6}$ primary CLL cells were activated by anti-human IgM coated streptavidin beads (M280) (Thermo Scientific) in $100 \mu \mathrm{l}$ media for 20 minutes. After an additional 30 minutes, OPP intensities were assessed by flow cytometry (Click-iT ${ }^{\mathrm{TM}}$ Plus OPP Alexa Fluor ${ }^{\mathrm{TM}} 488$ Protein Synthesis Assay Kit, Thermo Scientific). Mean fluorescence intensity (MFI) on FITC channel was calculated as representation of OPP labeling intensity. 


\section{Statistical analyses}

All experiments were repeated at least three times, and the means \pm SEM were calculated. The exact sample size for each experiment, biological or technical repeats are provided in the figure legends. Statistical analyses of results were performed using two-tail Student paired ttests. For experiments where more than two groups are compared, statistical analyses were performed using one-way ANOVA followed by two-tail Student paired t-tests. Statistical annotations as previously noted were denoted with asterisks according to the following, ${ }^{* * *} p$ $<0.001,{ }^{* *} p<0.01,{ }^{*} p<0.05$, and $n s p>0.05$.

\section{Data sharing}

RNA-seq data are available at GEO under accession number GSE149022. The mass spectrometry proteomics data have been deposited to the ProteomeXchange Consortium via the PRIDE partner repository ${ }^{15}$ with the dataset identifier PXD019465. Data not included in the main manuscript can be requested from the corresponding author.

Additional information can be found in Supplementary Data. 


\section{RESULTS}

Slow turnover of ZAP-70 in primary CLL cells requires extended in vitro culture for siRNA studies

The BCR-associated kinases Syk and ZAP-70 are presumed to share functional properties in transmitting signals in CLL cells ${ }^{6}$. Due to the high degree of homology between ZAP-70 and Syk, experiments employing small molecule inhibitors do not allow distinguishing between effects on ZAP-70 from those on Syk. To overcome these limitations, we knocked-down ZAP70 in primary CLL cells using RNA-interference (RNAi). (Patient characteristics are listed in Supplementary Table 8). Our initial experiments were unsuccessful and suggested that ZAP70 has a half-life of $>72$ hours. After extended ex vivo culture, however, we observed a significant down-regulation of ZAP-70 (Figures 1A,B). To maintain cell survival for further functional studies, cells were temporarily cultured on stroma cells, which substantially limited transfection-induced toxicity and spontaneous apoptosis of cells in suspension culture (Figure 1C). Importantly, RNAi with ZAP-70 expression did not affect Syk-expression, which allowed us to investigate ZAP-70 functions in primary CLL cells without the simultaneous inhibition of Syk (Figures 1D,E).

\section{ZAP-70 promotes cell survival but is not essential for proximal BCR-signaling}

Further down-stream analyses were performed on mono-culture by physically removing CLL cells from adherent stroma cells (Figure $2 A$ ), achieving pure lymphocyte populations of $>98 \%$ (data not shown). Since we were particularly interested in understanding how ZAP-70 modulates BCR-signaling, we first assessed whether culturing CLL cells on stroma cells affected the expression of surface IgM, which could possibly impinge on our experiments. Notably, surface lgM levels remained stable over time and were not affected by temporarily culturing cells on stroma cells (Figure 2B). Similarly, removal of CLL cells from stroma monolayers did not alter the expression of surface IgM (Figure 2C), indicating that our experimental system did not skew BCR signaling by modulating surface receptor expression. $\mathrm{BCR}$-activation with anti-IgM treatment rapidly induced $\mathrm{Ca}^{2+}$-flux, which unexpectedly was not altered by the protein levels of ZAP-70 (Figures 2D,E). We subsequently analyzed whether the presence of ZAP-70 affected the activation of proximal BCR-kinases following receptor activation by cross-linking IgM for 20 minutes. This stimulation caused strong phosphorylation of BLNK, Syk and PLC $\gamma 2$, which was not significantly different between ZAP-70 proficient and depleted cells, although we consistently detected a weaker phosphorylation of PLC $\gamma 2$ in the ZAP-70 knock-down cells (Figure 2F,G). Similarly, anti-IgM mediated AKT-activation was unaffected by the levels of ZAP-70 (Figure 2H). However, we observed a small, but consistent and significant difference in the baseline phosphorylation of AKT threonine 308, which is a 
known target for PDK1. The baseline phosphorylation of AKT serine 473, mediated by mTORC2, remained mostly unchanged by the expression of ZAP-70 (Figures $2 \mathrm{H}, \mathrm{I}$ ).

While proximal BCR signaling was largely unaffected by the expression of ZAP-70 in these experiments, extended in vitro culture demonstrated that the survival of CLL cells was significantly dependent on the presence of ZAP-70 in the absence of an induced BCR-signal (Figure 2J). Importantly, the reduced survival of ZAP-70 depleted CLL cells was rescued by IgM crosslinking (Figure $2 \mathrm{~K}$ ).

In conclusion, ZAP-70 expression in CLL cells is required for cell survival in the absence of an induced BCR-signal.

\section{ZAP-70 is required for the constitutive expression of CCL3, CCL4 and II4/1}

To further explore how ZAP-70 contributes to disease progression, we performed bulk RNAsequencing on primary CLL cells from two patients transfected with either ZAP-70 or control siRNA. The principal component analysis (PCA) of the raw gene abundances showed a clear separation between patients and ZAP-70 expression (Figure 3A). We reasoned that ZAP-70 dependent changes in gene expression could easily be masked by the enormous genetic heterogeneity of CLL samples ${ }^{16}$ (Figure $3 B$ ), and therefore initially used technical replicates on 2 patients, followed by validation-qPCR on additional samples. In total, we identified 40 differentially expressed (DE)-genes, with 27 commonly down-regulated and 13 up-regulated genes in ZAP-70-deficient samples (adjusted p-value $\left.<0.05, \log _{2}(F C)>0.5\right)$ (Figure $3 C$ and Supplementary Table 1). Importantly, MYC, CCL4 and CCL3 showed a significant downregulation by knocking-down ZAP-70 (Figure 3D). In addition, Cd1C and IL4I1, the latter contributing to immune-suppression in $\mathrm{CLL}^{17}$, were also downregulated by depleting ZAP-70 expression. ZAP-70 dependent expression of these genes was validated by qRT-PCR in additional ZAP-70 ${ }^{\text {pos }}$ patient samples (Figures 3E,F and Supplement Figure 1A). CCL3 and CCL4 are chemokines able to attract T cells and have previously been demonstrated to be regulated by $B C R$-signaling ${ }^{10}$. In line with this observation, inhibition of BTK with ibrutinib completely blunted the constitutive expression of CCL3 and CCL4 mRNA (Figure 3G).

Gene ontology (GO) enrichment analysis of the DE genes against the expression gene set also showed ZAP-70 involvement in 'cell migration' and 'antigen binding' (Figure $3 \mathrm{H}$ ). Importantly, BCR-receptor activation with anti-lgM treatment $(24 \mathrm{~h})$ did not significantly alter the ZAP-70 dependent gene expression profile (Supplement Figure S1B). Conclusively, these data provide evidence that ZAP-70 expression not only correlates with the expression of CCL3 and CCL4, but that it is actively involved in their constitutive gene expression. This regulation does not require additional triggering of a BCR-signal. 


\section{BCR-signaling enhances binding of ZAP-70 to ribosomes}

To gain further insights into the biology of ZAP-70 in CLL, we next isolated ZAP-70 immunecomplexes from primary CLL cells to identify binding-partners by mass spectrometry. For this, we employed the RIME (Rapid Immunoprecipitation Mass spectrometry of Endogenous proteins) protocol, which provides a sensitive method to identify protein complexes formed by endogenous proteins ${ }^{18}$. Proteins were double cross-linked with formaldehyde and the homobifunctional lysine cross-linker DSG prior to pull down. For the simultaneous analysis of cells either treated with anti-IgM or not, we combined RIME with isobaric labelling using Tandem Mass Tags (TMT-10plex) ${ }^{14}$, which allows for the quantitative comparison between replicates and conditions. These combined methods enabled us to detect and to quantify endogenous, transient protein complexes isolated from intact cells. We decided to first perform technical repeats from one patient given the enormous heterogeneity of CLL cells, followed by co-immunoprecipitation studies to validate binding partners on additional patient samples. The TMT-10plex-RIME raw data quantified 1476 unique proteins at a peptide false discovery rate (FDR) of $<0.01$. PCA showed a clear separation of ZAP-70 protein-complexes from IgG controls and between non-IgM and anti-lgM treated cells (Figure 4A). Expectedly, 20 minutes after anti-IgM treatment, binding of ZAP-70 to known bona-fide BCR signaling components was significantly enhanced (Figure 4B), indicating that this method reliably reports proteinbinding partners of ZAP-70. Comparison of IgG controls to proteins isolated from ZAP-70 complexes identified 1387 proteins with enhanced binding to ZAP-70 (Supplementary Table 2). Notably, we identified numerous BCR-related kinases constitutively bound to ZAP-70 in the absence of a triggered BCR-signal. Unexpectedly, a large proportion of bound proteins were ribosomal proteins, followed by proteins involved in metabolic processes and cell adhesion (Figures 4C,D). Further comparison of IgG controls to anti-lgM treated CLL cells showed an overall similar binding profile with a further enrichment of ribosomal proteins and BCR signaling proteins (Figures 4E,F and Supplementary Table 2). We then analyzed which proteins were differentially bound to ZAP-70 in the absence or presence of anti-lgM treatment and found enhanced binding of 214 proteins, while 162 proteins were less abundant after BCR stimulation (Figures 4G,H and Supplementary Table 2). Following BCR-activation, significantly more BCR-related kinases and ribosomal proteins were bound to ZAP-70.

\section{ZAP-70 binding to ribosomal proteins is associated with increased protein synthesis in CLL cells}

Cross-reactivity of the ZAP-70 antibody remained a major concern for us to discriminate 'noise' from true interacting proteins. To overcome this problem, we engineered the human Burkitt lymphoma B cell line BJAB to express FLAG-tagged full-length ZAP-70 fused to a BirA recognition peptide. BJAB cells, rather than MEC-1 cells, were chosen because they lack 
endogenous ZAP-70 expression (data not shown), which we considered a confounding factor in further experiments. Although BJAB cells are fundamentally different from primary CLL cells, we reasoned that the preserved dependency on BCR-signals ${ }^{19}$ for cell survival would allow to further investigate the function of ZAP-70 in B cells. For this BJAB-ZAP-70 cells were co-transfected with the E.coli derived biotin ligase BirA (thereafter named BJAB ${ }^{Z A P-70}$ ) to allow for in vivo biotinylation of ZAP-7020. Control cells were infected with the same constructs lacking the ZAP-70 coding region (Supplement Figure 2A). Surface IgM expression was not changed by expressing ZAP-70 (Supplement Figure 2B). Anti-IgM treatment of BJAB ZAP-70 or $B^{B} A^{\text {control }}$ cells induced calcium ${ }^{2+}$-flux without significant differences evoked by ZAP-70 expression (Supplement Figure 2C). Notably, slgM levels were generally higher than on CLL cells (data not shown), which may contribute to the absence of differences in calcium ${ }^{2+}-$ flux between ZAP-70 expresser and non-expresser. To identify proteins interacting with ZAP-70 in response to BCR-activation, cells were treated with anti-IgM for 20 minutes, prior to crosslinking with formaldehyde and labelling with TMT-10plex. In total, we identified 3015 unique proteins in the combined analysis (FDR<0.01). The PCA showed a clear separation of BJABcells expressing ZAP-70 or not and between anti-lgM treated and untreated cells (Supplement Figure 2D). Comparison of BJAB ${ }^{\text {control }}$ to $B J A B^{Z A P-70}$ cells in the absence of an induced BCRsignal identified 545 unique proteins constitutively bound in ZAP-70-complexes ( $p$ value $<0.05)$. Proteins known to mediate $B C R$-signals were identified with significantly enhanced binding after anti-IgM treatment (Supplement Figure 2E). Other detected proteins clustered in cell adhesion/ migration and metabolic processes (Supplement Figures 2F,G and Supplement Table 3). Importantly, nearly twice as many proteins were bound to ZAP-70 after BCR-stimulation compared to unstimulated cells (Supplement Figure $2 \mathrm{H}$ and Supplement Table 3). The majority of these newly recruited proteins belong to ribosomes (Supplement Figure 2I). Importantly, we performed mass-spectrometry on whole cell lysates from BJABZAP$70+/$ - anti-lgM treatment and did not find an enrichment of ribosomal proteins, indicating that increased binding to ZAP-70 is not related to a shift in the overall expression of those proteins (Supplement Figure 3A and Supplement Table 4).

Since primary CLL cells and BJAB cells shared substantial similarities in ZAP-70 proteincomplexes, we rationalized that combining mass spectrometry data obtained in CLL- and BJAB cells would be an opportunity to refine our analysis by further reducing the number of non-specifically bound proteins. For this, we subtracted unique proteins only found to be ZAP70 bound in either 'BJAB ${ }^{Z A P-70}$ or primary cells after BCR-stimulation. We thereby identified more than twice as many proteins $(n=147)$ interacting with ZAP-70 compared to unstimulated cells (Figure 5A and Supplement Table 5). Besides a strong enrichment of proteins essential for BCR-signaling (Figures 5B,C), a large proportion of these proteins were ribosomal proteins 
(Figures 5B-E). RPS-17 was one of the most enriched proteins in ZAP-70 complexes. Coimmunoprecipitation assays on additional patient samples confirmed the constitutive binding of ribosomal proteins to ZAP-70, further increased after BCR-activation (Figures 5F,G). Importantly, we did not observe binding of Syk to ribosomal proteins, suggesting that this property of ZAP-70 is not shared with Syk (Supplement Figure 3B). Interestingly, a recent study using light-affinity purification of ZAP-70 complexes from Jurkat cells also incidentally reported binding to ribosomes in T cells (Supplement Figure 4A) ${ }^{21}$.

Based on these results, we hypothesized that ZAP-70 is recruited to ribosomes in response to BCR-activation. To generate further evidence for this, we sought to demonstrate colocalization of ZAP-70 to ribosomal proteins by applying a method not relying on cross-linkage of proteins. For this, we performed polysome profile analysis, which employs ultracentrifugation of cell lysates over a sucrose gradient to separate different ribosome units from total cell lysates. Quantification of RNA by spectrophotometry in each of the sucrosefraction indicated that the separation of ribosomes was successful (Figure $5 \mathrm{H}$ ). We detected few polyribosomes, possibly related to the quiescent state of cells and altered ribosome maturation in CLL compared to normal B cells, as reported previously 22 . Following BCRreceptor activation with anti-IgM we observed a significant increase of ZAP-70, co-migrating with the 60-80 ribosome fraction and to a lesser extent in the polyribosome fraction (Figure $5 \mathrm{I}$, J).

We hypothesized that BCR-induced binding of ZAP-70 to ribosomal proteins could have effects on translation. To test this, we applied O-propargyl-puromycin (OP-Puro) to assess the total level of protein synthesis in unstimulated and in anti-IgM activated, primary cells, differing only in their expression of ZAP-70. Metabolic labelling demonstrated that protein synthesis was significantly reduced in CLL cells depleted of ZAP-70 (Figure 5K). Since many of its functions depend on kinase activity, we hypothesize that ZAP-70 mediates phosphorylation of ribosomal proteins. The hypothesis that ZAP-70 is a RBP-modifying kinase is further supported by previously published experiments in $\mathrm{T}$ cells: Employing a modified kinase domain of ZAP-70, tyrosine modifications of 15 ribosomal proteins were observed within minutes after inhibition of ZAP-70 in activated Jurkat T cells (Supplement Figure 4B) ${ }^{23}$. The kinetics of these phosphorylation-changes suggest that ZAP-70 may function, either directly or indirectly, as a ribosomal protein kinase, with the net effect of promoting protein biosynthesis.

A previous study has identified MYC being a target for translational regulation in CLL and increased by BCR-stimulation ${ }^{24}$. We therefore tested whether ZAP-70 levels could impact on MYC expression in CLL cells. Interestingly, constitutive MYC-levels were significantly reduced in ZAP-70 depleted cells (Figures 5L,M). In contrast, BCR-mediated upregulation of MYC was unaffected by ZAP-70 levels, indicating again that ZAP-70 levels are critical for the regulation 
of biological processes in the absence of an induced BCR-signal (Figure $5 \mathrm{~N}$ and Supplement Figure 4C).

\section{DISCUSSION}

Nearly two decades ago ZAP-70 expression was identified in malignant B cells in a subset of CLL patients ${ }^{4}$, possessing strong prognostic value for an aggressive disease course ${ }^{5}$. Since then, several studies have demonstrated that ZAP-70 can enhance BCR-signaling ${ }^{8}$, though kinase-activity was dispensable for this ${ }^{6,7}$. This suggested that ZAP-70 acts predominantly as an adaptor protein, promoting phosphorylation of other BCR signaling components, such as Syk $^{6}$. Our data further advance our understanding of the biology of ZAP-70 in CLL by demonstrating biological functions which do not depend on a strong BCR signal. In contrast to previously published work ${ }^{7}$, we have not observed significant, ZAP-70 dependent changes in calcium-flux or the activation of proximal BCR-kinases following receptor ligation with antiIgM. A possible explanation for this apparent conundrum is that in our experiments the residual ZAP-70 activity after (an incomplete) knockdown of the protein may fully compensate. The observed moderate, but statistically not significant decrease in PLC $\gamma 2$-phopsphorylation would support this idea. However, our experimental approach also differs significantly from others, which have overexpressed ZAP-70 in negative, IGHV mutated CLL cells ${ }^{7}$. Therefore, it remains possible that the described BCR signaling-enhancing effects of ZAP-70 are more prominent in intrinsically ZAP-70 negative cells, predominantly harboring mutated IGHV genes ${ }^{5}$ and differing in the cell-of-origin ${ }^{25}$.

Regardless of these differences, which require further experimental work to clarify, we identified that ZAP-70 constitutively contributes to cell survival, protein synthesis and gene expression in the absence of an overt, strong BCR-signal. The dependency of these effects on ZAP-70 varied between patients in our experiments, which is likely related to substantial variations in the baseline expression of ZAP-70 among positive-patients, siRNA-mediated degree of ZAP-70 depletion, presence of chromosomal abnormalities and IGHV mutational status. Larger studies are needed to systematically address whether ZAP-70 has more or less pronounced affects in any of these subgroups.

The constitutive binding of ZAP-70 to BCR-signaling kinases as well as the moderate, but statistically significant decrease in AKT activation associated with decreased expression of ZAP-70 suggest that ZAP-70 could enhance a tonic BCR-signal. The constitutive activation of $\mathrm{PI3K}$ in CLL cells supports this idea and may contribute to the baseline activation of $A K T^{26}$. However, activation of alternative, pro-survival signaling pathways must also be considered.

Our data demonstrate that the depletion of endogenous ZAP-70 not only negatively affected cell survival, but also the expression of genes known to be important for the recruitment and 
interaction with T cells. CCL3 and CCL4 are both chemokines able to attract CD4 ${ }^{+} \mathrm{T}$ cells, which can further induce CLL proliferation through the activation of CD40. In vivo evidences supporting this hypothesis derive from high CCL3 plasma levels found in ZAP-70 positive patients, associated with a shorter time to first treatment ${ }^{27}$. Furthermore, increased CCL3 expression in CLL lymph nodes correlate with $\mathrm{KI} 67^{+}$expression and number of $\mathrm{CD}^{+} \mathrm{T}$ cells ${ }^{28}$. Our data further advance these correlative findings and demonstrate that ZAP-70 actively contributes to the expression of these chemokines and suggest that ZAP-70 positive cells can create a more favorable, disease-promoting microenvironment in the absence of a strong BCR-signal.

Another, unexpected finding from our experiments was the identification of protein complexes between ZAP-70 and ribosomal proteins, particularly enhanced upon BCR activation. This observation begs the question of its functional implications. Previous proteomic analyses have described distinct profiles between ZAP-70pos UM- and ZAP-70neg M-CLL; these differences were constitutively present while BCR-activation only increased further changes in ZAP-70 UM-CLL but not in M-CLL ${ }^{29}$. In context of these results, our data indicate that protein-binding to ZAP-70 is modified by BCR-signals and suggest that it may contribute to these differences in global protein expression through transcriptional and post-transcriptional mechanisms.

Our data are in keeping with a recent report, showing that BCR-signaling drives mRNA translation, partly through $M Y C^{24}$. We here identified ZAP-70 as an enhancing factor for this. De-regulation of protein biosynthesis in CLL has also been reported by the Willis group, who identified significantly reduced ribosomal maturation and activity related to reduced translation of ribosomal RNA ${ }^{22}$. However, no subtype analyses were performed dependent on ZAP-70 status. A more recent, extensive proteomic characterization of CLL cells identified 'RNAprocessing' and 'RNA-binding' as major discriminators between malignant and non-malignant $B$ cells, though these findings were not restricted to a particular subset of patients ${ }^{30}$. Lastly, mutations in RPS15, found in a fraction of patients, cause alterations in translation initiation and fidelity, associated with global protein changes ${ }^{31}$. In conclusion, alterations in proteinbiosynthesis are a common finding in CLL and a result of distinct underlying mechanisms. Our data identify ZAP-70 as another factor contributing to de-regulated protein synthesis. It remains unknown from our work how exactly ZAP-70 promotes mRNA translation. Since we used double-cross linking to identify binding-partners, we cannot specify which protein(s) are essential for its association with ribosomes. The possibility that ZAP-70 directly interacts with ribosomal proteins is supported by ZAP-70 associated changes in tyrosine-phosphorylation of ribosomal proteins observed in activated $\mathrm{T}$ cells ${ }^{23}$. Post-translational modifications of ribosomal proteins have been shown to modulate protein synthesis ${ }^{32,33}$, though the precise mechanisms are poorly defined. We also consider other possibilities of ZAP-70 binding to ribosomes, such as binding to RNA or through functioning as a scaffold protein bound to newly 
synthesized proteins. An alternative, though not mutually exclusive, possibility is that ZAP-70 regulates protein synthesis throughs its effects on MYC expression, since MYC itself can promote translation through direct and indirect mechanisms ${ }^{34,35}$.

In conclusion, our data show that ZAP-70 is directly involved in the regulation of gene expression and protein synthesis, with the net-effect of improved cell survival. Therefore, ZAP70 may increase disease aggressiveness through its function as a coordinating signaling node in malignant $B$ cells, for which a strong BCR-signal is not essential.

\section{AKNOWLEDGEMENTS}

We would like to express our gratitude to all patients from Addenbrooke's outpatient clinic that provided blood samples for this research. The help of Alison Wray and Joanna Baxter to recruit patients to this study was essential for our work. We are very grateful for our discussions with Art Weiss, who provided ZAP-70 constructs and also valuable feedback and suggestions for future experiments. We also thank Daniel Hodson for carefully reading the manuscript, his scientific input and the provision of BJAB-BirA cells. We appreciate constructive discussions of our results with Martin Turner. A.J.W. was supported by a Specialist Programme from Blood Cancer UK (12048, to AJW), the UK Medical Research Council (MR/T012412/1), the Kay Kendall Leukaemia Fund, a Wellcome Trust strategic award to the Cambridge Institute for Medical Research (100140), a core support grant from the Wellcome Trust and MRC to the Wellcome Trust-Medical Research Council Cambridge Stem Cell Institute, the Connor Wright Project, the Cambridge National Institute for Health Research Biomedical Research Centre and the European Cooperation in Science and Technology (COST) Action CA18233 "European Network for Innovative Diagnosis and treatment of Chronic Neutropenias, EuNet INNOCHRON". This research was supported by the Kay Kendell Foundation (KKLF, grant KKL1070) and by Cancer Research UK (CRUK) (CRUK; C49940/A17480) to I.R.; I.R. is a senior CRUK fellow.

\section{AUTHORSHIP CONTRIBUTION}

J.C., V.S., A.M., C.J., S.T. performed wet-lab experiments, analyzed data and created figures. RNA-seq data were analyzed by A.S. and I.M.. Proteomic experiments were planned in close collaboration with C.D'S. and analyzed by V.N.R.F. and C.S.R.C., who also provided raw data and figures. The study was planned by I.R. with guidance of S.B.H and A.W.. I.R. wrote the manuscript. 


\section{DISCLOSURE OF CONFLICTS OF INTEREST}

All authors declare no conflict of interest. I.R. has previously received a research grant from

AstraZeneca and advised the company as a consultant, both activities are irrelevant to this work. 


\section{REFERENCES}

1. Iwashima M, Irving BA, Oers NS van, Chan AC, Weiss A. Sequential interactions of the TCR with two distinct cytoplasmic tyrosine kinases. Science (New York, N.Y.). 1994;263(5150):1136-1139.

2. Wang $\mathrm{H}$, Kadlecek TA, Au-Yeung BB, et al. ZAP-70: an essential kinase in T-cell signaling. Cold Spring Harbor perspectives in biology. 2010;2(5):a002279-a002279.

3. Crespo M, Bosch F, Villamor N, et al. ZAP-70 expression as a surrogate for immunoglobulin-variable-region mutations in chronic lymphocytic leukemia. The New England journal of medicine. 2003;348(18):1764-1775.

4. Wiestner A, Rosenwald A, Barry TS, et al. ZAP-70 expression identifies a chronic lymphocytic leukemia subtype with unmutated immunoglobulin genes, inferior clinical outcome, and distinct gene expression profile. Blood. 2003;101(12):4944-4951.

5. Rassenti LZ, Huynh L, Toy TL, et al. ZAP-70 compared with immunoglobulin heavy-chain gene mutation status as a predictor of disease progression in chronic lymphocytic leukemia. The New England journal of medicine. 2004;351(9):893-901.

6. Gobessi S, Laurenti L, Longo PG, et al. ZAP-70 enhances B-cell-receptor signaling despite absent or inefficient tyrosine kinase activation in chronic lymphocytic leukemia and lymphoma B cells. Blood. 2007;109(5):2032-2039.

7. Chen L, Huynh L, Apgar J, et al. ZAP-70 enhances IgM signaling independent of its kinase activity in chronic lymphocytic leukemia. Blood. 2008;111(5):2685-2692.

8. Chen L, Widhopf G, Huynh L, et al. Expression of ZAP-70 is associated with increased Bcell receptor signaling in chronic lymphocytic leukemia. Blood. 2002;100(13):4609-4614.

9. Mockridge $\mathrm{Cl}$, Potter KN, Wheatley I, et al. Reversible anergy of slgM-mediated signaling in the two subsets of CLL defined by VH-gene mutational status. Blood. 2007;109(10):44244431.

10. Burger JA, Quiroga MP, Hartmann E, et al. High-level expression of the T-cell chemokines CCL3 and CCL4 by chronic lymphocytic leukemia B cells in nurselike cell cocultures and after BCR stimulation. Blood. 2009;113(13):3050-3058.

11. Richardson SJ, Matthews C, Catherwood MA, et al. ZAP-70 expression is associated with enhanced ability to respond to migratory and survival signals in B-cell chronic lymphocytic leukemia (B-CLL). Blood. 2006;107(9):3584-3592.

12. Calpe $E$, Codony $C$, Baptista MJ, et al. ZAP-70 enhances migration of malignant $B$ lymphocytes toward CCL21 by inducing CCR7 expression via IgM-ERK1/2 activation. Blood. 2011;118(16):4401-4410.

13. Calpe E, Purroy N, Carpio C, et al. ZAP-70 promotes the infiltration of malignant Blymphocytes into the bone marrow by enhancing signaling and migration after CXCR4 stimulation. PloS one. 2013;8(12):e81221. 
14. Papachristou EK, Kishore K, Holding AN, et al. A quantitative mass spectrometry-based approach to monitor the dynamics of endogenous chromatin-associated protein complexes. Nature communications. 2018;9(1):2311.

15. Perez-Riverol Y, Csordas A, Bai J, et al. The PRIDE database and related tools and resources in 2019: improving support for quantification data. Nucleic Acids Res. 2018;47(D1):gky1106-.

16. Puente XS, Pinyol M, Quesada V, et al. Whole-genome sequencing identifies recurrent mutations in chronic lymphocytic leukaemia. Nature. 2011;475(7354):101-105.

17. Sadik A, Patterson LFS, Öztürk S, et al. IL4I1 Is a Metabolic Immune Checkpoint that Activates the AHR and Promotes Tumor Progression. Cell. 2020;182(5):1252-1270.e34.

18. Mohammed H, Taylor C, Brown GD, et al. Rapid immunoprecipitation mass spectrometry of endogenous proteins (RIME) for analysis of chromatin complexes. Nature protocols. 2016;11(2):316-326.

19. Roit FD, Engelberts PJ, Taylor RP, et al. Ibrutinib interferes with the cell-mediated antitumour activities of therapeutic CD20 antibodies: implications for combination therapy. Haematologica. 2014;100(1):haematol.2014.107011.

20. Roux KJ, Kim DI, Raida M, Burke B. A promiscuous biotin ligase fusion protein identifies proximal and interacting proteins in mammalian cells. The Journal of cell biology. 2012;196(6):801-810.

21. Hörner M, Eble J, Yousefi OS, et al. Light-Controlled Affinity Purification of Protein Complexes Exemplified by the Resting ZAP70 Interactome. Frontiers in immunology. 2019;10:226.

22. Sbarrato T, Horvilleur E, Pöyry T, et al. A ribosome-related signature in peripheral blood CLL B cells is linked to reduced survival following treatment. Cell death \& disease. 2016;7(6):e2249-e2249.

23. Goodfellow HS, Frushicheva MP, Ji Q, et al. The catalytic activity of the kinase ZAP-70 mediates basal signaling and negative feedback of the $T$ cell receptor pathway. Science signaling. 2015;8(377):ra49-ra49.

24. Yeomans A, Thirdborough SM, Valle-Argos B, et al. Engagement of the B-cell receptor of chronic lymphocytic leukemia cells drives global and MYC-specific mRNA translation. Blood. 2016;127(4):449-457.

25. Seifert M, Sellmann L, Bloehdorn J, et al. Cellular origin and pathophysiology of chronic lymphocytic leukemia. The Journal of experimental medicine. 2012;209(12):2183-2198.

26. Ringshausen I, Schneller F, Bogner C, et al. Constitutively activated phosphatidylinositol-3 kinase (PI-3K) is involved in the defect of apoptosis in B-CLL: association with protein kinase Cdelta. Blood. 2002;100(10):3741-3748.

27. Sivina M, Hartmann E, Kipps TJ, et al. CCL3 (MIP-1 $\alpha$ ) plasma levels and the risk for disease progression in chronic lymphocytic leukemia. Blood. 2011;117(5):1662-1669. 
28. Hartmann EM, Rudelius M, Burger JA, Rosenwald A. CCL3 chemokine expression by chronic lymphocytic leukemia cells orchestrates the composition of the microenvironment in lymph node infiltrates. Leukemia \& lymphoma. 2016;57(3):563-571.

29. Perrot $A$, Pionneau $C$, Nadaud $S$, et al. A unique proteomic profile on surface IgM ligation in unmutated chronic lymphocytic leukemia. Blood. 2011;118(4):e1-15.

30. Johnston HE, Carter MJ, Larrayoz M, et al. Proteomics Profiling of CLL Versus Healthy B-cells Identifies Putative Therapeutic Targets and a Subtype-independent Signature of Spliceosome Dysregulation. Molecular \& cellular proteomics : MCP. 2018;17(4):776-791.

31. Bretones G, Álvarez MG, Arango JR, et al. Altered patterns of global protein synthesis and translational fidelity in RPS15-mutated chronic lymphocytic leukemia. Blood. 2018;132(22):2375-2388.

32. Miller JL, Cimen H, Koc H, Koc EC. Phosphorylated proteins of the mammalian mitochondrial ribosome: implications in protein synthesis. Journal of proteome research. 2009;8(10):4789-4798.

33. Soung GY, Miller JL, Koc H, Koc EC. Comprehensive analysis of phosphorylated proteins of Escherichia coli ribosomes. Journal of proteome research. 2009;8(7):3390-3402.

34. Schmidt EV. The role of c-myc in regulation of translation initiation. Oncogene. 2004;23(18):3217-3221.

35. Lin C-J, Cencic R, Mills JR, Robert F, Pelletier J. c-Myc and elF4F Are Components of a Feedforward Loop that Links Transcription and Translation. Cancer Res. 2008;68(13):53265334. 


\section{FIGURE LEGENDS}

Figure 1. SiRNA effectively depletes ZAP-70 without affecting Syk expression in primary CLL cells.

A. Representative ZAP-70 and $\beta$-actin immunoblots of primary CLL cells at indicated time points post siRNA nucleo-transfection with a non-specific siRNA and ZAP-70 siRNA.

B. Quantification of ZAP-70 expression using ImageJ(C) and calculated as ratio (siZAP70/NSC) after normalization to $\beta$-actin signal. Time points indicate time after nucleofection; $(n=3)$.

C. Percentage of live (Annexin V-, DAPI-) CLL cells on day 7 of transfection. Primary CLL samples $(n=7)$ were transfected with NSC or ZAP70 siRNA and cultured for 7 days on stroma cells.

D. Representative immunoblots of ZAP-70, Syk and $\beta$-actin of primary, purified CLL cells 7 days post siRNA transfection.

E. Quantification of ZAP-70 and Syk expression, normalized to $\beta$-actin, 7 days post siRNA transfection; $(n=7)$.

Statistical analyses of results were performed using one-way analysis of variance (ANOVA), following paired two-tailed Student t-tests. ns, not significant. ${ }^{*} p<5 \times 10^{-2} ;{ }^{* *} p<10^{-2}$. 
Figure 2. ZAP-70 contributes to cell survival, but is not essential for IgM-stimulated BCR-signaling

A. Scheme shows the strategy for siRNA knock-down of ZAP-70 in primary ZAP-70 positive CLL cells and co-culture on feeder cells. Down-stream analyses were performed on purified CLL cells in mono-culture. Created with BioRender.com

B. Mean Fluorescence Intensity (MFI) of cell surface IgM on CD19+ CLL cells. Primary ZAP70 positive CLL cells $(n=5)$ were mono-cultured or co-cultured on stromal cells for 1 or 2 days before assessment of surface IgM by FACS analysis.

C. Graph shows the Mean Fluorescence Intensity (MFI) of cell surface IgM on CD $19^{+} \mathrm{CLL}$ cells transfected with non-specific siRNA or ZAP-70 siRNA. Primary ZAP-70 positive CLL cells $(n=6)$ were transfected with siRNA and co-cultured with stromal cells for 7 days and then separated from stromal cells for additional 24 hours in mono-culture.

D. Representative kinetic plots show the calcium flux of one primary CLL sample after transfection with NSC or ZAP-70 siRNA. CLL cells were harvested and labeled with Fluo4 (FITC) after 24 hours in mono-culture. Anti-lgM activation was triggered 30 seconds after flow cytometric measurement started.

E. Quantification of calcium-flux response of CLL samples $(n=7)$ transfected with NSC or ZAP-70 siRNA. Ratios were calculated by using kinetic plots, dividing peak Fluo-4 (FITCA) intensity induced by anti-IgM activation by baseline Fluo-4 intensity.

F. Representative phospho-BLNK (Tyr96), phospho-Syk (Tyr525/526), phospho-PLC $\gamma 2$ (Tyr759), total BLNK, total Syk, total PLC $\gamma 2$, ZAP-70 and $\beta$-actin immunoblots of primary CLL cells mono-cultured for 24 hours after non-specific siRNA or ZAP-70 siRNA transfection. To induce BCR-signaling, mono-cultured CLL cells were treated with beadsbound anti-lgM for 20 minutes.

G. Quantification of phospho-kinases relative to total (unphosphorylated) proteins after 24 hours in mono-culture after siRNA transfection, using Image JC software $(n=6)$.

H. Representative phospho-AKT (Ser473), phospho-AKT (Thr308), total AKT and $\beta$-actin immunoblots of primary CLL cells mono-cultured for 24 hours after non-specific siRNA or ZAP-70 siRNA transfection. For anti-IgM stimulated samples, mono-cultured CLL cells were treated with beads-bound anti-lgM for 20 minutes. [SE=short exposure].

I. Quantification of phospho-AKT relative to total (unphosphorylated) AKT after 24 hours in mono-culture after siRNA transfection, using ImageJC software $(n=4$ or 5 , as indicated).

J. Percentage of live (Annexin $\left.\mathrm{V}^{-}, \mathrm{DAPl}^{-}\right) \mathrm{CLL}$ cells, cultured for 48 or 72 hours in monoculture post transfection ( $n=10$ and $n=8$, respectively).

K. Percentage of live (Annexin $\mathrm{V}^{-}$, DAPI-) CLL cells, cultured in mono-culture post transfection $(n=7)$ and treated with anti-lgM for 48 hours $(n=6)$. 


\section{Figure 3: ZAP-70 constitutively regulates gene expression relevant for $\mathrm{T}$ cell interactions}

A. Principal component analysis (PCA) created on the top 2000 most abundant genes, illustrates the separation across patients (mainly captured in PC1) and treatments (PC2).

B. Venn Heatmap representing the Jaccard Similarity Index (JSI), on the top 2000 most abundant genes, across all pairwise comparisons on patients and treatments. The JSI spans the 0 to 1 range, which corresponds to no overlap (0) to identical sets of genes (1). The clear separation between patients is contrasted by closer similarity on replicates and treatments.

C. Volcano blot illustrating the DE genes (x-axis presents the log2(FC) between the Control and si70 samples; $y$-axis presents the -log10(p-value)); grey points correspond to non-DE genes, red points correspond to genes for which the $p$-value $<0.05$ (but $|\log 2(F C)|<0.5$ ), blue points are the DE genes called this analysis, color-intensity is proportional to the log2 of the average normalized expression levels across the compared samples.

D. Heat map of genes from two individual primary patients CLL cells treated with NSC or ZAP-70 siRNA. Red and blue indicate relatively high and low expression, respectively. Each condition analyzed depicts technical replicates, derived from 2 individual siRNA transfections. Genes were ranked by average Log2FC from EdgeR analysis from bottom to top.

E. QRT-PCR analysis of CD1C and IL4I1 in primary CLL cells (CD19+-positive selected) ( $\mathrm{n}=8$ and $n=7$ respectively) transfected with non-specific siRNA or ZAP-70 siRNA and further mono-cultured for 24 hours. Relative gene expression level is normalized to reference gene GAPDH and compared to NSC control.

F. QRT-PCR analysis of CCL3 and CCL4 in primary CLL cells (CD19+-positive selected) $(n=8$ and $n=7$, respectively) transfected with non-specific siRNA or ZAP-70 siRNA and further mono-cultured for 24 hours. Relative gene expression level is normalized to reference gene GAPDH and compared to NSC control.

G. QRT-PCR analysis of CCL3 and CCL4 in primary CLL cells (CD19+positive selected) $(n=4)$ transfected with non-specific siRNA or ZAP-70 siRNA. Ibrutinib was treated at $0.5 \mu \mathrm{M}$ for 24 hours before cell harvesting. Relative gene expression level is normalized to reference gene GAPDH and compared to NSC control.

$\mathrm{H}$. Gene ontology (GO) enrichment analysis of the DE-genes in (C). Background genes are the set of all genes that pass the noise threshold. 


\section{Figure 4: Constitutive binding of ZAP-70 to BCR-kinases and ribosomal proteins}

A. Principal component analysis (PCA) of protein abundance, representing the variability between anti-ZAP-70 pull down -/+ anti-IgM stimulation for 20 minutes and IgG isotype control. Each dot represents one technical replicate with an individual pull-down of ZAP70 or $\lg$.

B. Heat map of proteins annotated in KEGG 'B cell receptor signaling pathway', identified by mass spectrometry as ZAP-70 binding partners. Red and blue indicate relatively high and low protein abundance, respectively. Each condition analyzed depicts three (unstimulated) or four (IgM activated) technical replicates. Proteins were ranked from left to right by Log2FC ratio comparing IgM activated to unstimulated group.

C. Volcano plot showing proteins bound to ZAP-70 (Log2FC>0) vs. IgG isotype control $($ Log2FC $<0)$ in unstimulated CLL cells.

D. Gene set enrichment analysis (GSEA) was applied to identify pathways that ZAP-70 associated proteins are associated with in unstimulated CLL cells. Pathways are listed in order of Normalized Enrichment Scores (NES) in columns (top x-axis). FDR-q values for each pathway set are indicated by the red dotted line (lower x-axis). Colors of column represent different sub-grouped cell functions.

E. Volcano plot showing proteins bound to ZAP-70 (Log2FC>0) vs. IgG isotype control $($ Log2FC $<0)$ in anti-lgM activated CLL cells.

F. Gene set enrichment analysis (GSEA) to identify pathways associated with ZAP-70 in antiIgM activated CLL cells.

G. Volcano plot showing proteins differentially bound to ZAP-70 (Log2FC $>0)$ in unstimulated vs. anti-lgM activated states.

$\mathrm{H}$. Gene set enrichment analysis (GSEA) to identify pathways associated with ZAP-70 after BCR-activation with in anti-lgM. 


\section{Figure 5. BCR-activation induces binding of ZAP-70 to ribosomal proteins and is associated with enhanced protein-biosynthesis}

A. Venn diagram comparing ZAP-70-binding proteins identified by mass spectrometry (MS) in anti-IgM activated (20 minutes) primary CLL cells and BJAB cells (Log2FC>1, adj $p<0.01$ ).

B. GSEA gene ontology (GO) terms extracted from the unique 147 proteins identified in (A) from both primary CLL cells and BJAB cells. GO signatures are listed in order of Normalized Enrichment Scores (NES) in columns (top x-axis). FDR-q values for each signature set are indicated by the red dotted line (lower $\mathrm{X}$-axis). Colors of column represent different sub-grouped cell functions.

C. The 147 unique proteins from $(A)$ were plotted by the Log2 fold change (Log2FC). X-axis, identified in primary CLL cells MS; Y-axis, identified in BJAB cells. Colors indicate the subgrouped functions of the proteins. Grey dots indicate proteins involved in other cellular functions.

D. Heat map of ZAP-70-associated ribosomal proteins from unstimulated or anti-IgM activated primary CLL cells. (adjusted $p<0.05$ ). Red and blue indicate relatively high and low protein abundance, respectively. Each row analyzed depicts a technical replicate.

E. Heat map of ZAP-70-associated ribosomal proteins from unstimulated or anti-lgM activated $B J A B^{Z A P-70}$ cells versus non-specifically biotinylated proteins from $B J A B^{\text {control }}$ cells (adjusted $p<0.05$ ). Red and blue indicate relatively high and low protein abundance, respectively. Each row analyzed depicts a technical replicate.

F. Whole cell lysates from primary CLL cells, unstimulated or stimulated with anti-lgM for 20 minutes, were immunoprecipitated (IP) with a ZAP-70 antibody or control IgG and immunoblotted (IB) with antibodies for ZAP-70, Syk and RPS17.

G. Quantification of RPS17 bound to ZAP-70. The levels of RPS17 co-immunoprecipitated with ZAP-70 were normalized to total ZAP-70 levels. Anti-IgM treated samples were then normalized to non-treated samples $(n=4)$.

$\mathrm{H}$. Comparison of polysome profiles between unstimulated (blue) and anti-lgM activated (20 minutes) (red) primary CLL samples. Vertical lines separating the curves indicate the fractions mainly extracted from 40 s ribosome subunits, 60-80s ribosome subunits and polysomes.

I. Proteins from sucrose gradient fractions in primary CLL cells were precipitated by $20 \%$ (w/v) trichloroacetic acid (TCA) and immunoblotted with ZAP-70, RPL29 and RPS17. Blue boxed graphs: unstimulated cells; Red boxed graphs: anti-IgM activated cells. Fractions 6-19 represent 40 s subunits, $60-80$ s subunits, polysomes, separated by vertical lines.

J. Quantification of relative ZAP-70 protein levels (relative to RPL17) in indicated fractions in primary CLL samples $(n=3)$. Anti-lgM activated samples were normalized to unstimulated samples.

K. Graph showing the relative (to untreated NSC) fold change in OPP labeling (30 minutes) in unstimulated or anti-lgM activated (20') primary CLL cells $(n=11)$, previously transfected with NSC or ZAP-70 siRNA.

L. Representative MYC and $\beta$-actin immunoblots of primary CLL cells mono-cultured for 24 hours after non-specific siRNA or ZAP-70 siRNA transfection.

M. Quantification of c-MYC levels in primary CLL cells proficient or depleted of ZAP-70. Graphs were generated using ImageJ (C) $(n=4)$.

N. Representative immunoblots for MYC, ZAP-70 and $\beta$-actin of primary CLL cells monocultured for 24 hours after non-specific siRNA or ZAP-70 siRNA transfection. To induce BCR-signaling, mono-cultured CLL cells were treated with bead-bound anti-lgM for 24 hours. 
A

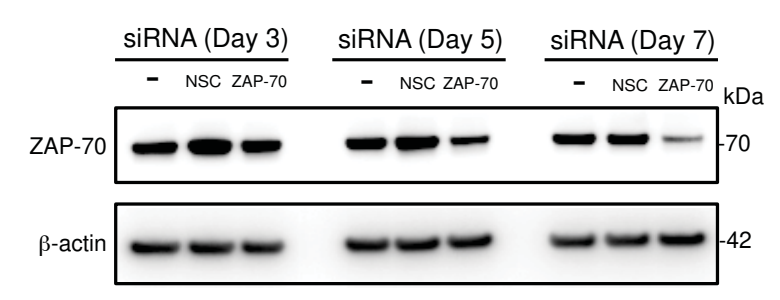

D

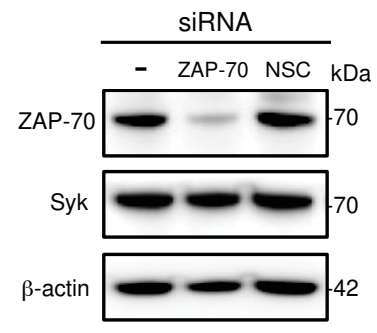

E
B

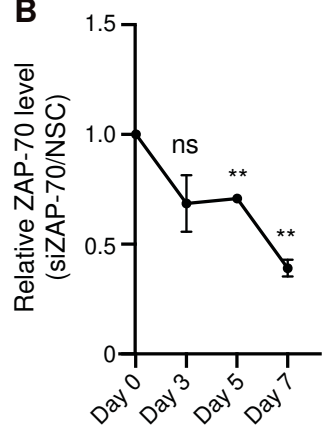

C

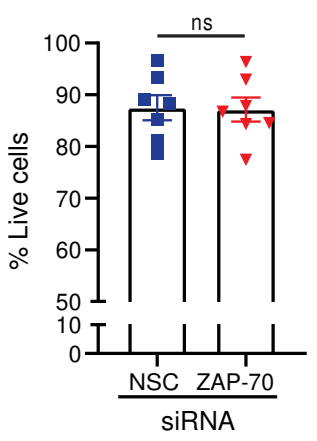

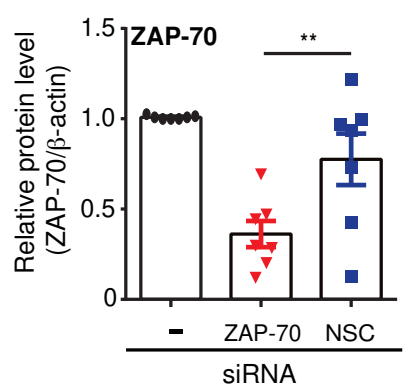

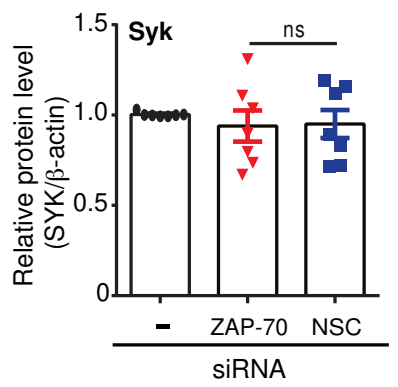

Figure 1 Chen et.al 

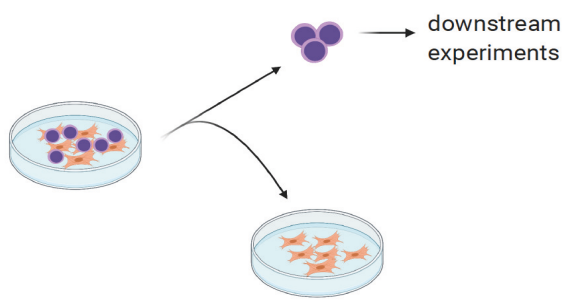

MSCs
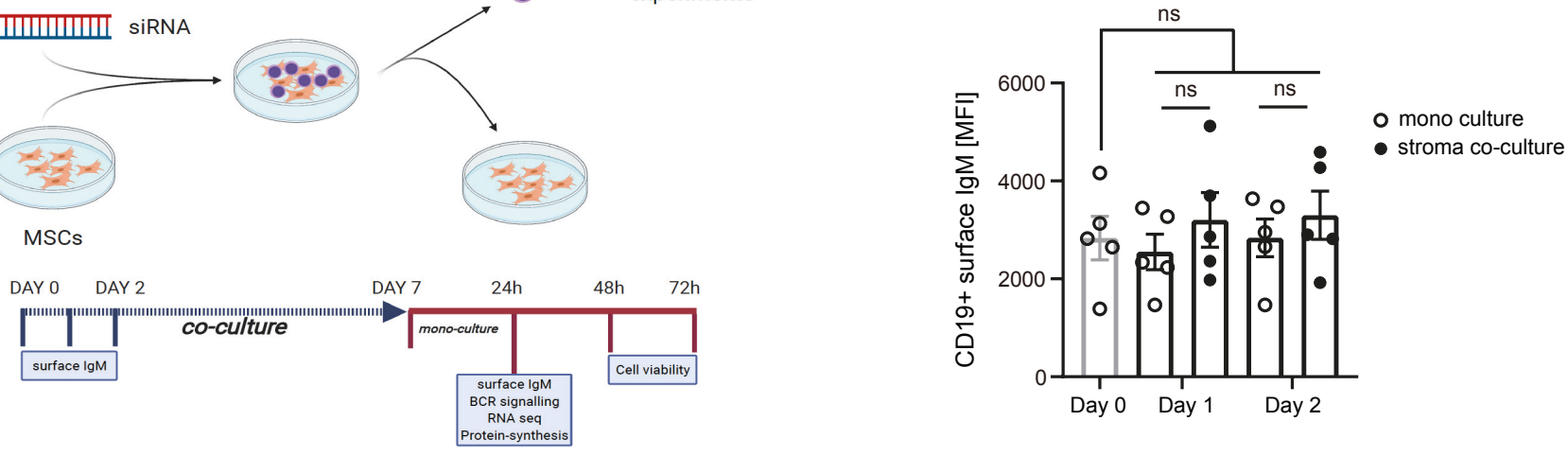

C

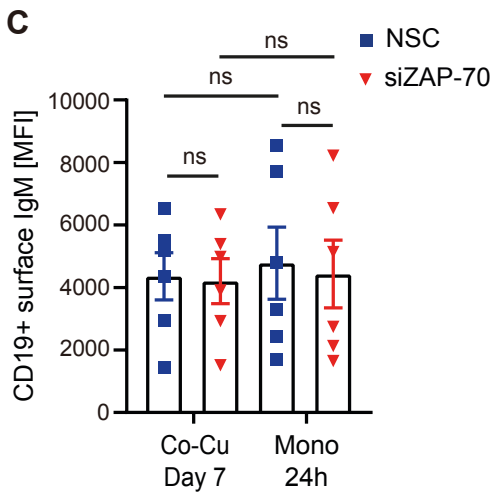

F

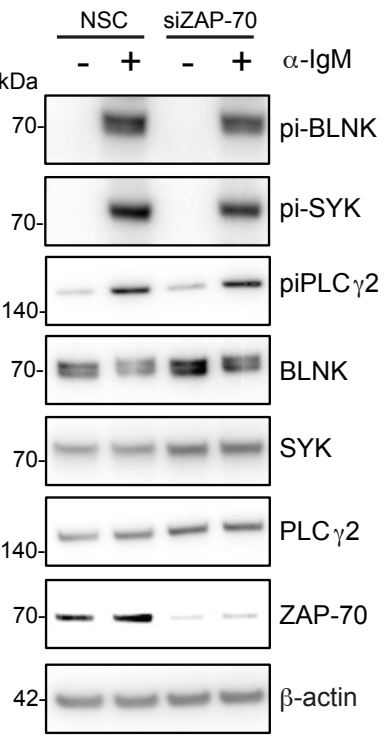

J

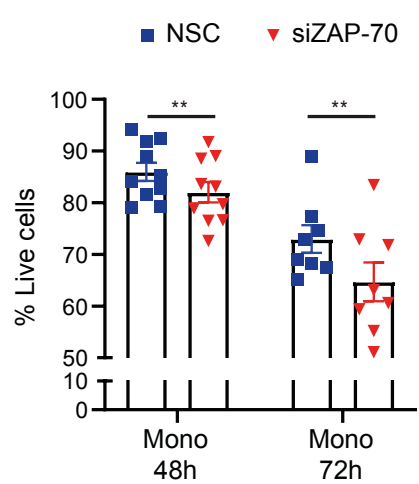

D

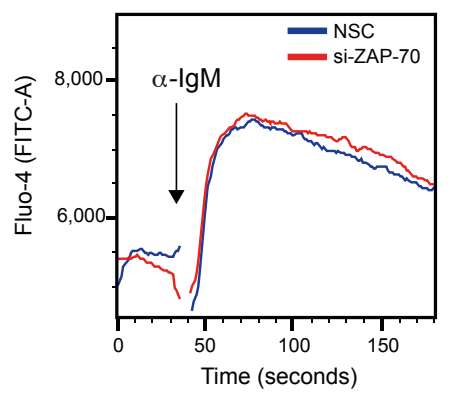

E

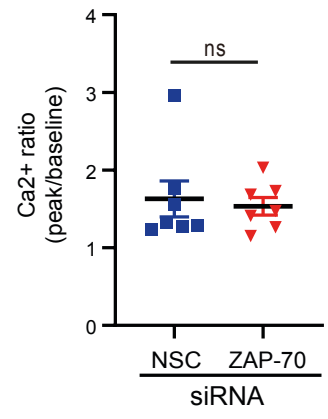

G

口 NSC $\nabla$ siZAP-70 (unstimulated)

- NSC $\nabla$ siZAP-70 (anti-lgM)
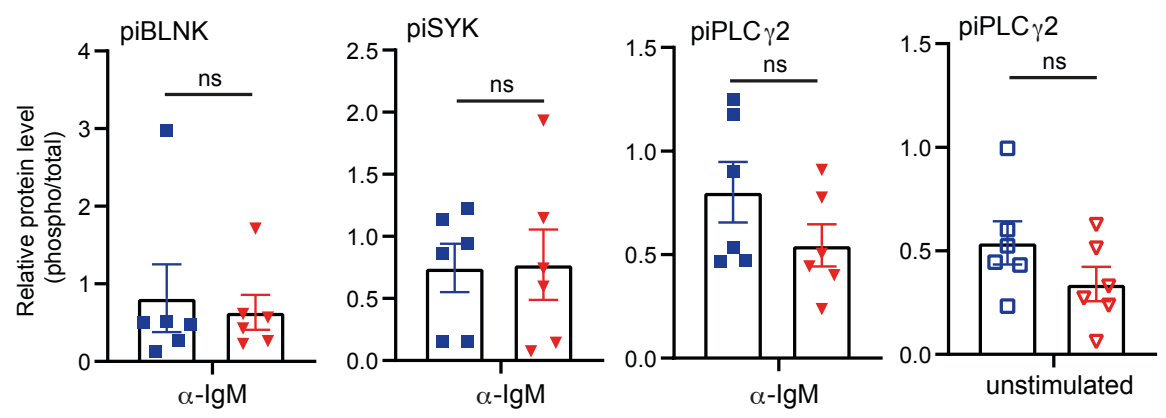

H
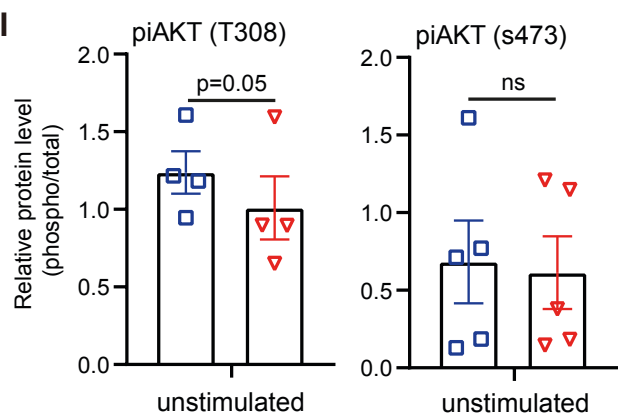

K

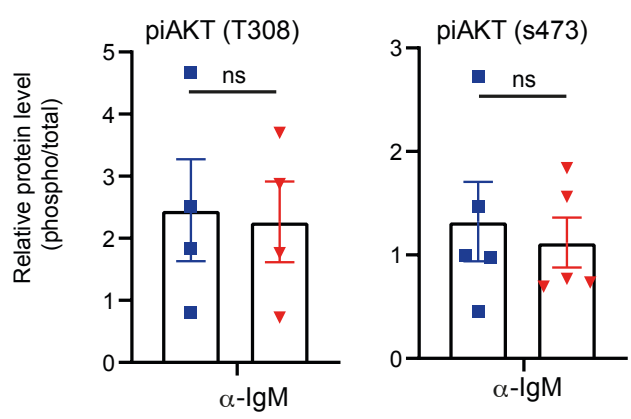

- NSC

v siZAP-70

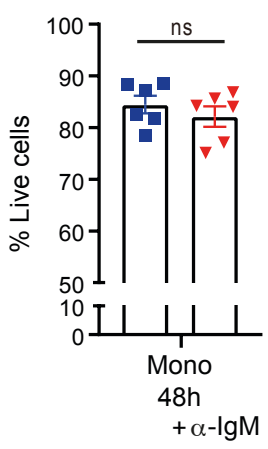


A

RNAseq

(2000 most abundant genes)

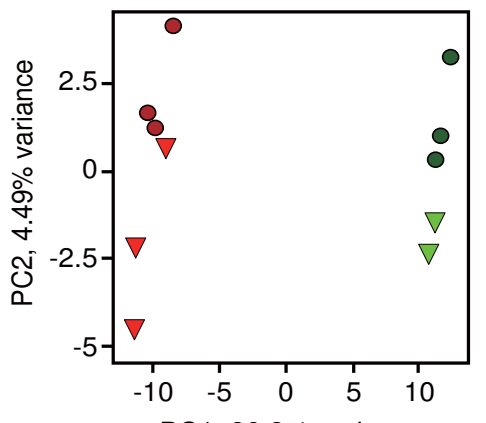

$\mathrm{PC} 1,90.8 \%$ variance

oNSC,patient1 $\quad \nabla$ siZAP-70,patient1 oNSC,patient2 $\nabla$ siZAP-70,patient2

C

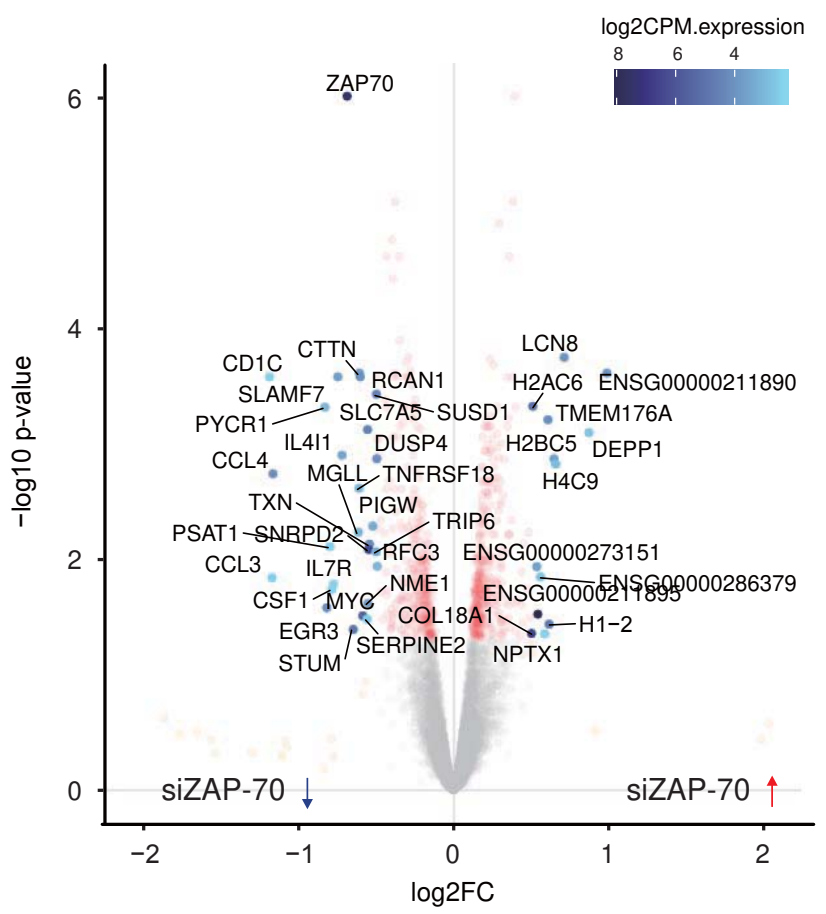

B

(2000 most abundant genes)

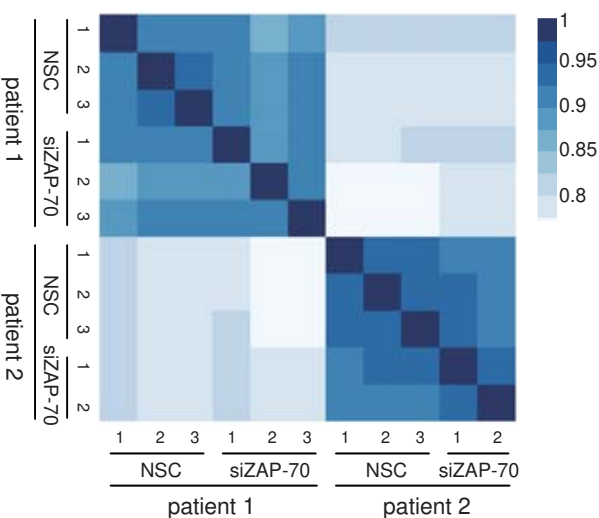

D

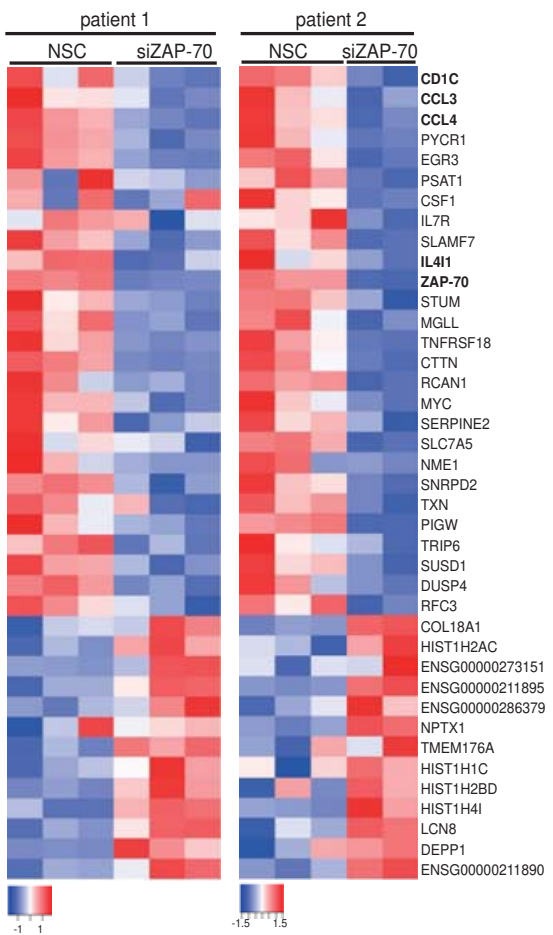

E

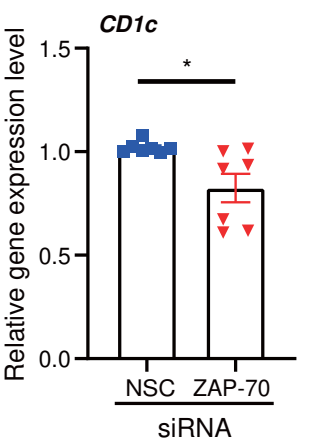

G

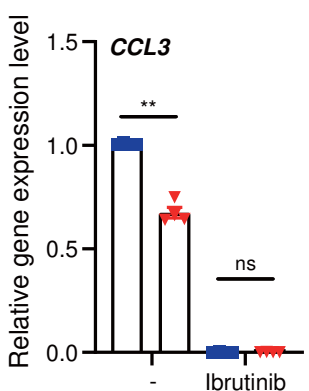

$\mathbf{F}$
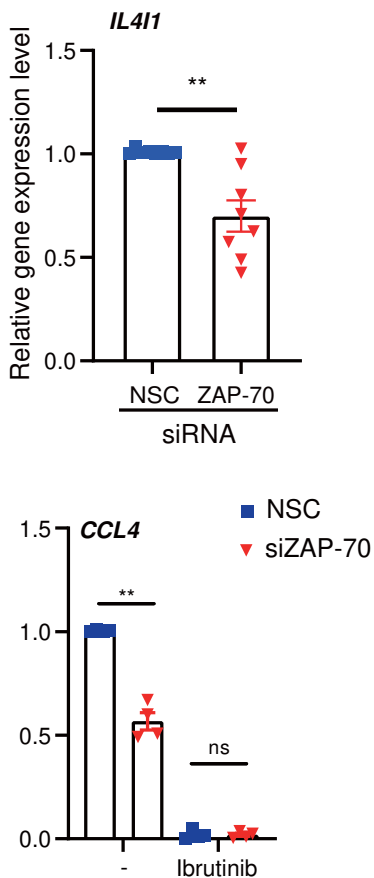
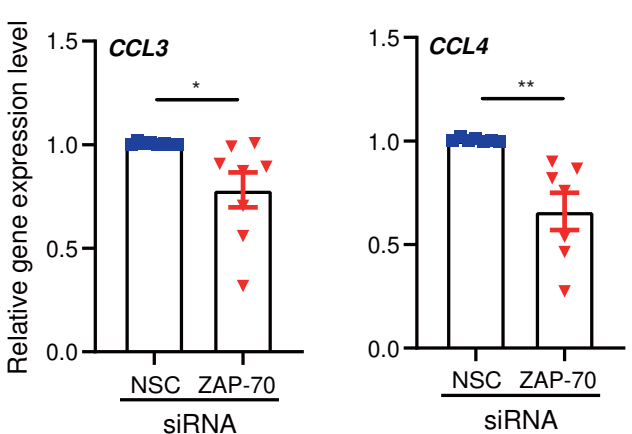

- NSC

$\checkmark$ siZAP-70

H

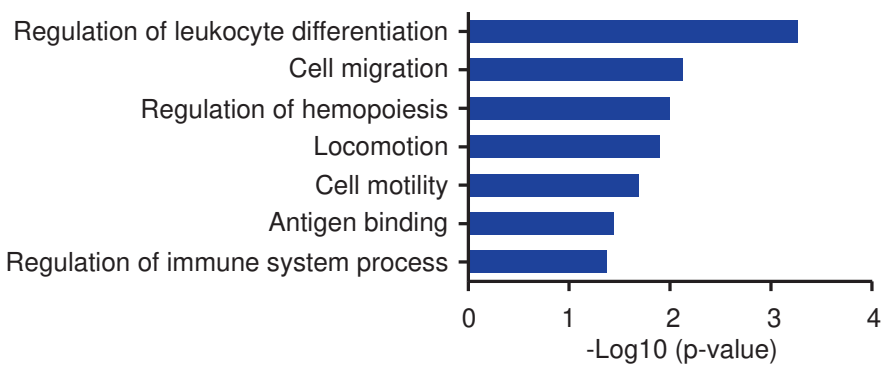




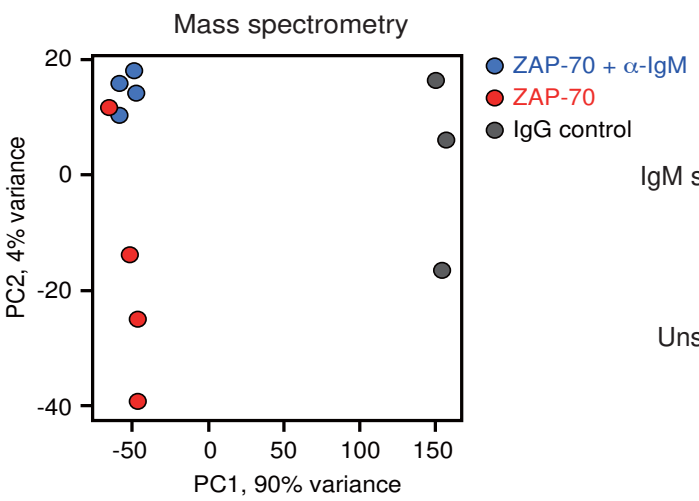

C

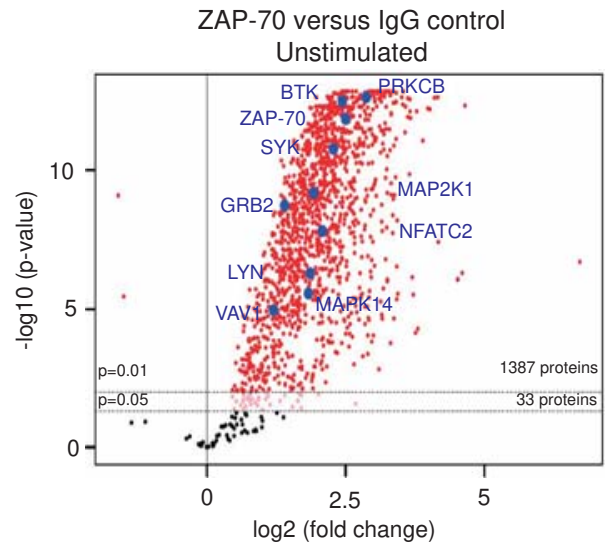

E

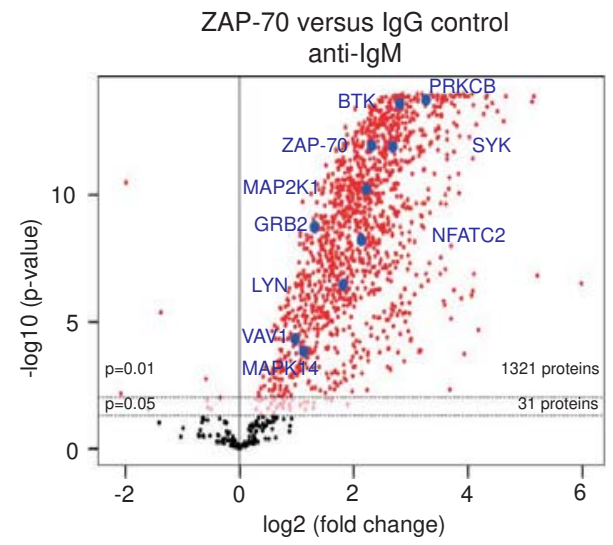

G

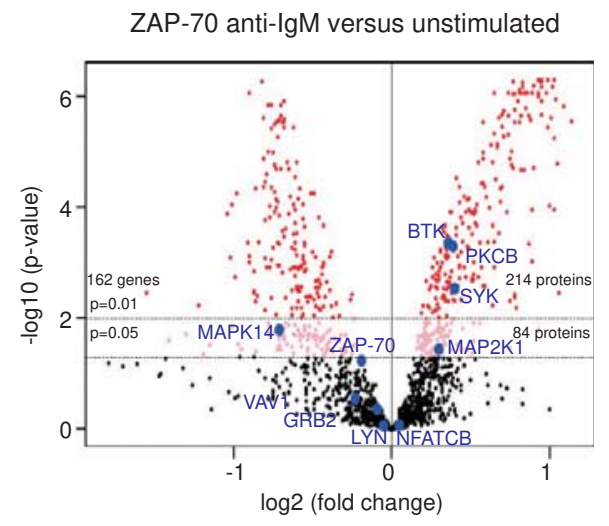

B cell receptor signaling pathway
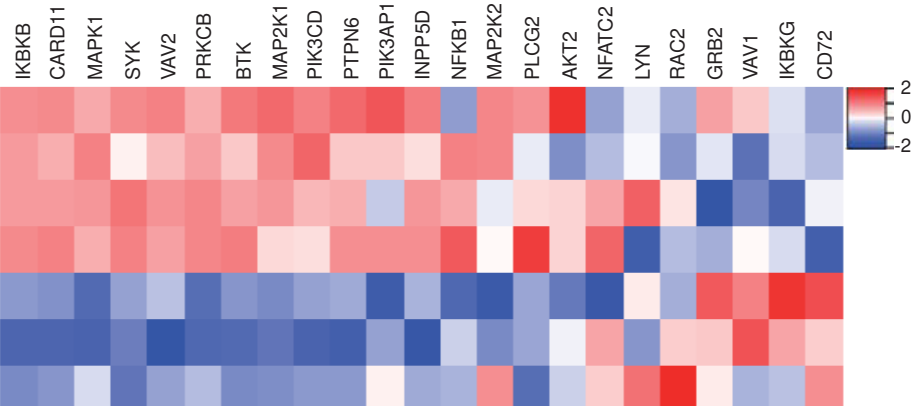

D

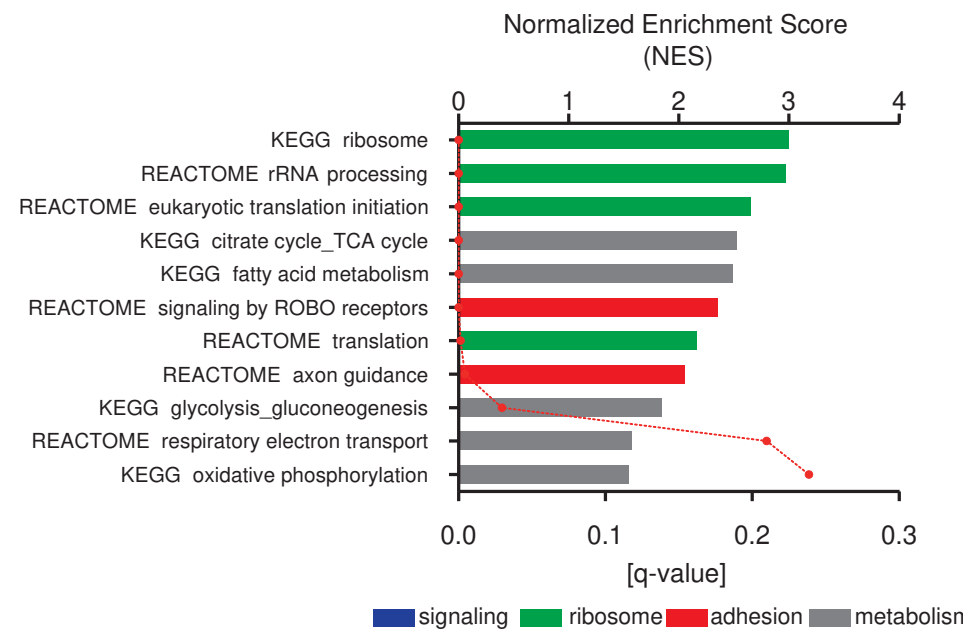

$\mathbf{F}$

Normalized Enrichment Score

(NES)

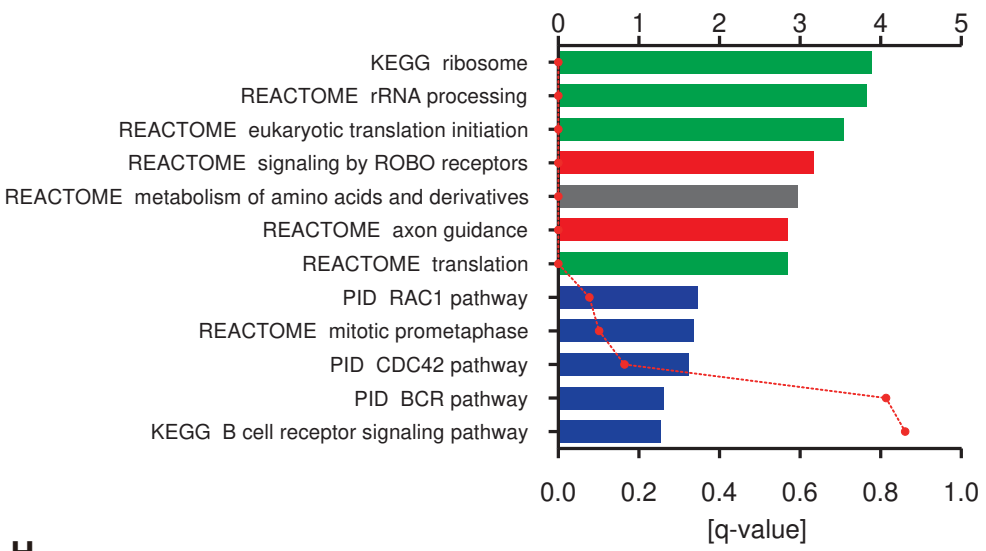

Normalized Enrichment Score

(NES)

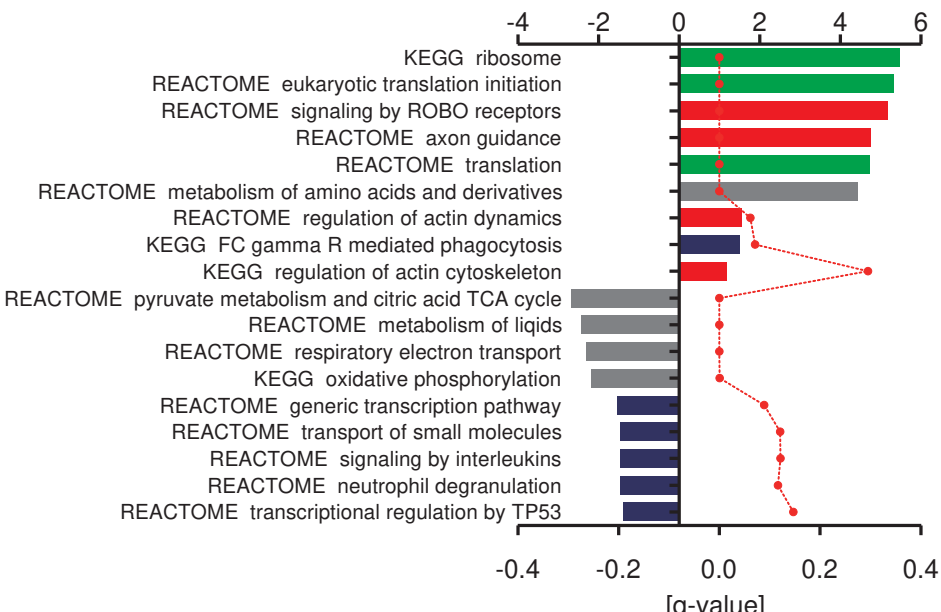

Figure 4

Chen et al 
A

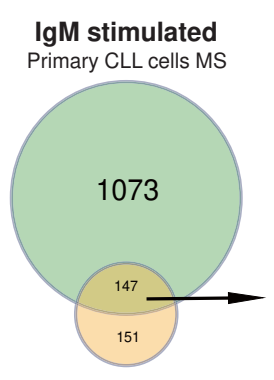

BJAB cell line MS
B

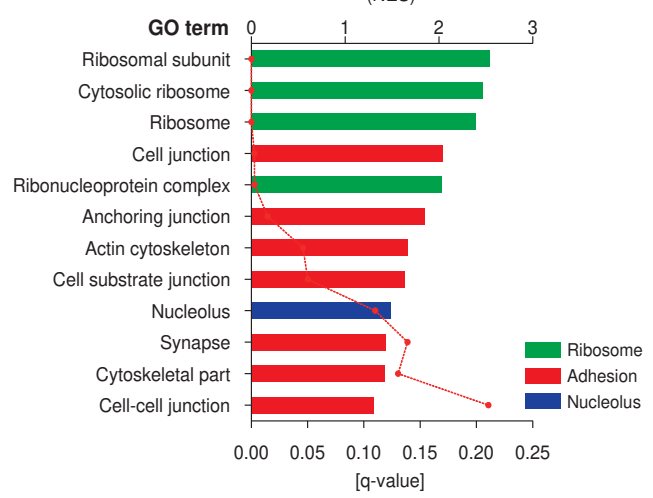

E

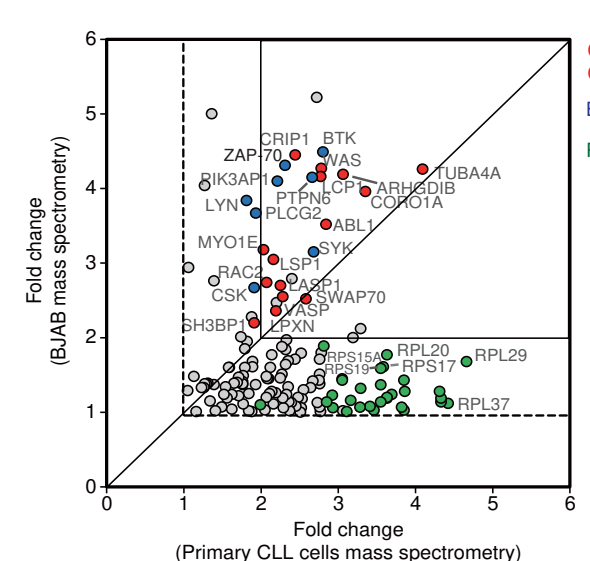

$F$
Primary CLL cells

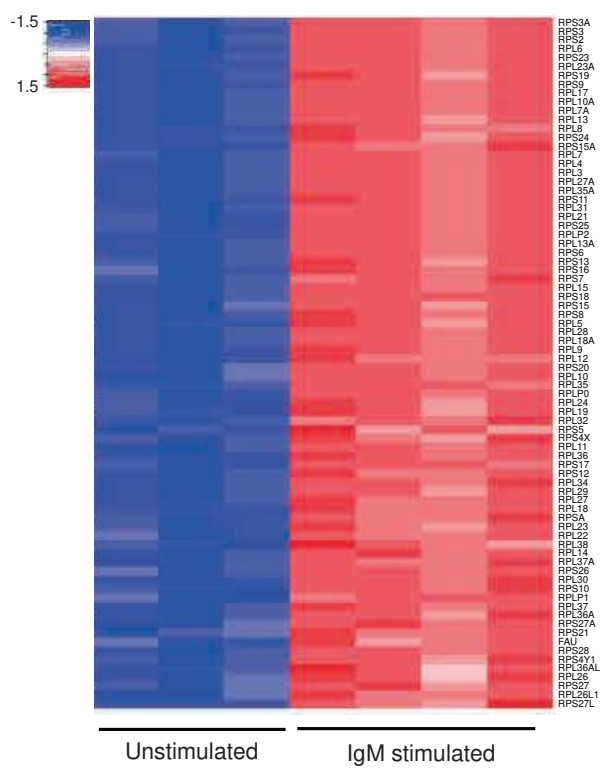

H

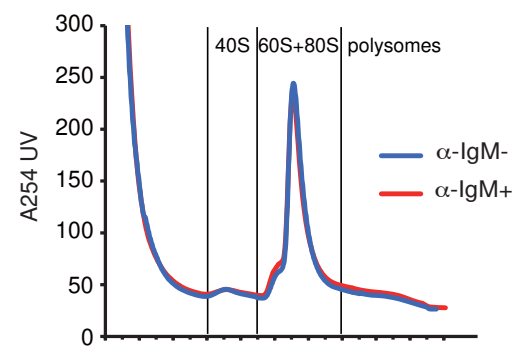

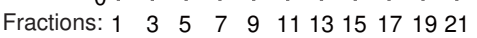
$5 \%-45 \%$ sucrose gradient

K

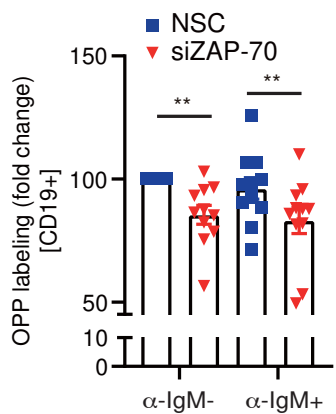

BJAB cell line
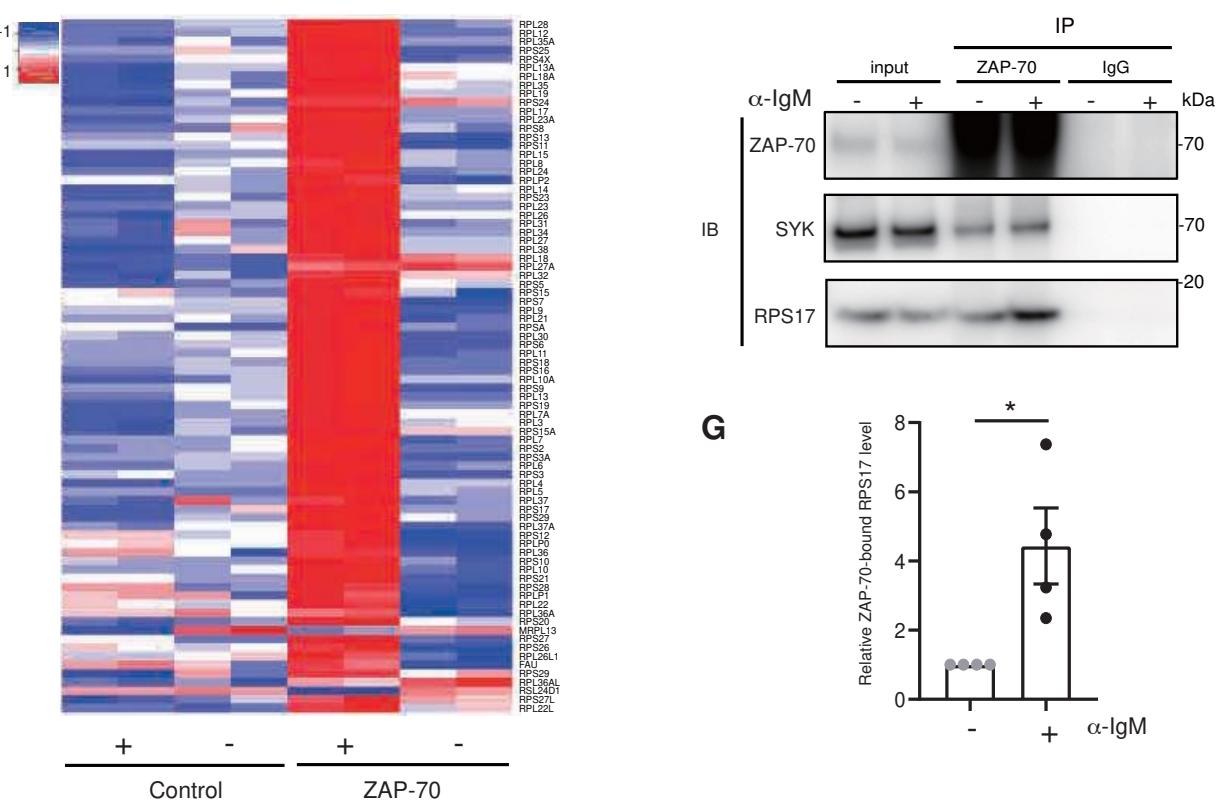

G

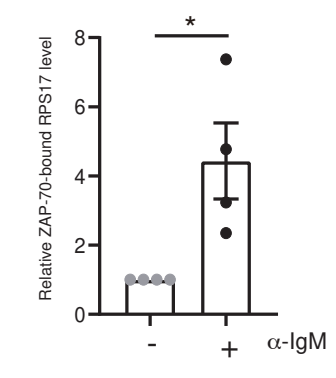

N

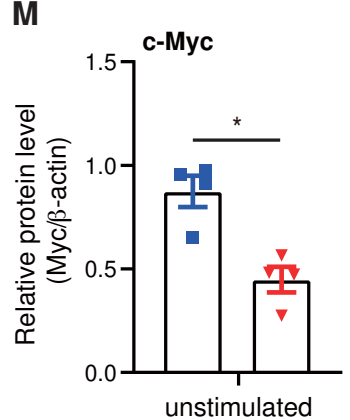

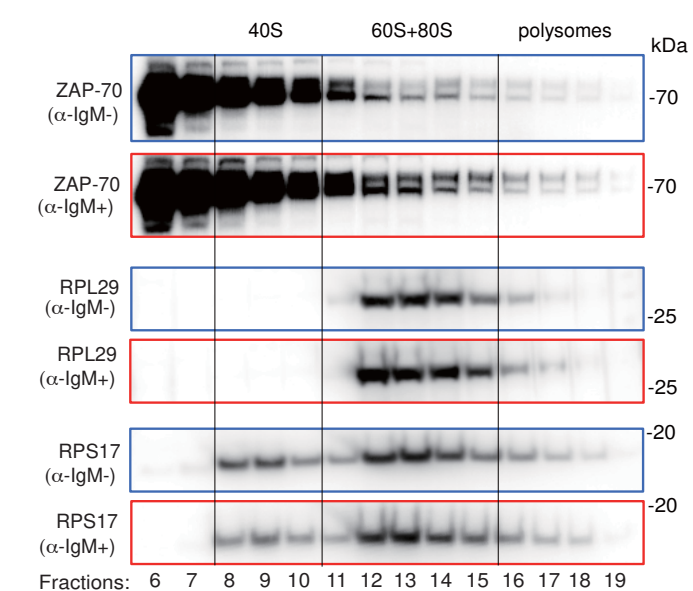

J

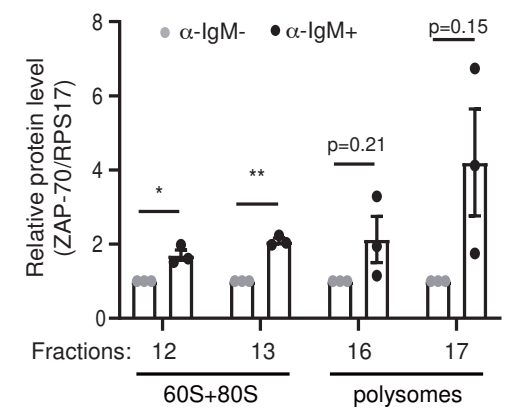

Cytoskeleton binding, Cell adhesion \& migration BCR signaling Ribosomal proteins

Figure 5

Chen et.al 